\title{
Isatuximab plus pomalidomide/ dexamethasone versus pomalidomide/ dexamethasone in relapsed/refractory multiple myeloma: ICARIA Phase III study design
}

\author{
Paul G Richardson ${ }^{*}, 1$, Michel Attal ${ }^{2}$, Frank Campana ${ }^{3}$, Solenn Le-Guennec ${ }^{4}$, Ai-Min \\ Hui $^{\ddagger}{ }^{\prime}$, Marie-Laure Risse ${ }^{4}$, Kathryn Corzo ${ }^{3}$ \& Kenneth C Anderson ${ }^{1}$
}

'Medical Oncology, Dana-Farber Cancer Institute, Harvard Medical School, Boston, MA 02215-5450, USA

${ }^{2}$ Department of Hematology, Institut Universitaire du Cancer Toulouse Oncopole, Toulouse, France

${ }^{3}$ Sanofi, Cambridge, MA 02142, USA

${ }^{4}$ Sanofi, Vitry-Alfortville, France

*Author for correspondence: paul_richardson@dfci.harvard.edu

*Employee of Sanofi when this publication was developed.

Treatment for relapsed/refractory multiple myeloma (RRMM) remains an unmet need. Isatuximab, an anti-CD38 monoclonal antibody has shown efficacy and tolerability as a monotherapy and combination therapy in Phase I/II studies in RRMM. Here, we describe the design of the Phase III ICARIA-MM study (NCT02990338) which will evaluate isatuximab in combination with pomalidomide (Pom) and low-dose dexamethasone (dex) (Pom/dex) versus Pom/dex alone in RRMM. Patients will be randomized in a 1:1 ratio. The primary endpoint is progression-free survival. Response will be determined by an independent response review committee using IMWG criteria (2016) and safety will be assessed throughout. Approximately 300 patients (150 in each arm) are expected to enroll. The first patient was recruited in January 2017 and accrual is ongoing.

First draft submitted: 10 November 2017; Accepted for publication: 12 December 2017; Published online: 22 December 2017

Keywords: $\mathrm{CD} 38 \cdot$ immunomodulatory drugs $\bullet$ monoclonal antibody $\bullet$ multiple myeloma • progression-free survival $\cdot$ protocol $\bullet$ trial in progress

Multiple myeloma (MM) is a malignant plasma cell disease, characterized by proliferation of plasma cells in the bone marrow (BM) and the production of excessive amounts of a monoclonal immunoglobulin. MM is predominantly associated with advancing age, with $>80 \%$ of patients aged $\geq 60$ years [1]. Patients with MM experience bone pain/fractures, fatigue, anemia, infections, hypercalcemia and renal dysfunction.

The disease course for MM varies in its aggressiveness and prognosis for each patient. Certain chromosomal abnormalities in $\mathrm{MM}$ are associated with poor clinical outcomes, including high-risk cytogenetic changes such as del(17p), $\mathrm{t}(4 ; 14), \mathrm{t}(14 ; 16), \mathrm{t}(14 ; 20)$ and gain(1q) $[2,3]$. Initial MM treatment options are based upon the patient's age, fitness and disease status, and involve chemotherapeutic agents, in combination with stem cell transplant when patients are eligible; to achieve deep anti-MM effects [4].

Novel therapeutic agents include immunomodulatory drugs (IMiDs) such as thalidomide, lenalidomide (Len) or pomalidomide (Pom) and proteasome inhibitors (PIs) such as bortezomib, carfilzomib or ixazomib [5-10]. These agents have demonstrated better outcomes in patients with MM,

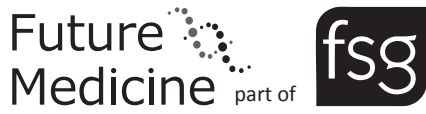


including higher response rates and lower toxicity, compared with standard, nonspecific chemotherapy as induction therapy [5,11-13]. PIs act through multiple mechanisms to suppress tumor survival pathways and to arrest tumor growth, tumor spread and angiogenesis [14]. IMiDs increase T-cell and natural killer (NK) cell activation, induce apoptosis and inhibit cell adhesion molecules [15].

Pom is a second-generation IMiD, and is approved for use in combination with low-dose dexamethasone (dex) in patients with MM who have received $\geq 2$ lines of therapy, including Len and a PI (USA only) or bortezomib (Europe), and have progressed on their last line of therapy [16]. The development of PI and/or IMiD-based regimens has significantly improved clinical outcomes for patients with MM, with median survival increasing from 3 to 6 years over the past two decades $[1,9,17,18]$. However, patients who fail the current standard of care treatments, including PIs and IMiDs, have a poor prognosis, and new treatments are necessary $[19,20]$.

Novel agents approved for the treatment of MM include the monoclonal antibodies (mAbs) daratumumab (DARA) and elotuzumab (ELO) which target cell surface molecules highly expressed on MM cells [21-25]. DARA targets CD38, a type II transmembrane glycoprotein that functions both as a signal-transducing receptor and a multifunctional ectoenzyme. The expression of CD38 is increased in $\mathrm{MM}$, and $>90 \%$ of the malignant plasma cells from patients with MM show surface expression of CD38 [26]. The strong expression of CD38 on malignant MM cells contrasts with the expression pattern on normal cells, supporting its selection as a target antigen [26]. DARA received initial approval as monotherapy in patients with heavily pretreated RRMM who were refractory to PIs and IMiDs [23]. DARA has since been approved in combination with Pom/dex, Len/dex and bortezomib/dex for the treatment of RRMM [27].

ELO targets SLAMF7, a glycoprotein expressed on MM and NK cells, but not on normal cells, and demonstrated potent anti-MM activity in a Phase III study comparing ELO plus Len/dex with Len/dex alone in patients with RRMM [21]. This study led to the approval of ELO in combination with Len/dex in patients with MM who had received 1-3 prior therapies. The studies of ELO and DARA have demonstrated the potent effects of $\mathrm{mAb}$ therapies against MM, supporting the development of additional agents in this class and, in particular, testing their potential activity in patients with heavily pretreated and refractory disease [28].

\section{Isatuximab}

Isatuximab (ISA) is an IgG1 $\kappa \mathrm{mAb}$ that binds selectively to a unique epitope on CD38 and targets tumor cells through a combination of mechanisms of action including antibody-dependent cellular cytotoxicity, antibody-dependent cellular phagocytosis, complement-dependent cytotoxicity and immune cell depletion/inhibition of immunosuppressive cells [22,29] (Figure 1). Preclinical assessment suggests that NK cell-mediated antibody-dependent cellular cytotoxicity is the most important mechanism of action contributing to ISA efficacy [30]. In addition, ISA appears to be unique among anti-CD38 mAbs, in that it is able to induce apoptosis without crosslinking and may be better tolerated, with a shorter infusion time [22,31]. Furthermore, ISA demonstrated significant inhibition of CD38 ectoenzyme activity in MM and other hematologic cancer cell lines [32]. In preclinical studies, ISA inhibited CD38 ectoenzyme activity ten-times more potent than an anti-CD38 mAb constructed using the published peptide sequence of DARA [33], however the clinical implications of this have not yet been determined. CD38 is highly expressed on regulatory T cells (Tregs) in patients with MM. Tregs expressing CD38 at high levels are elevated in the circulation of patients with hematologic malignancies [29], which has been shown to correlate with tumor burden and disease progression in other types of cancer [34,35]. ISA inhibits the suppressive function of Tregs by promoting apoptosis and inhibiting proliferation of these cells. Targeting CD38 with ISA may therefore induce immunomodulatory effects, which both relieve immunosuppression and trigger anti-MM immunity [29]. Treating MM cells or Tregs with IMiDs, such as Pom or Len, has been shown to increase the expression of CD38 levels on the surface of these cells [29,36].

In addition to demonstrating the single-agent anti-MM activity of ISA, preclinical studies have shown that ISA cell killing activity is significantly enhanced when combined with Len/dex or Pom/dex, with the greatest increase in anti-MM activity observed when ISA was combined with Pom/dex [36]. These preclinical results supported the investigation of ISA as a monotherapy and in combination with IMiDs in clinical studies. 


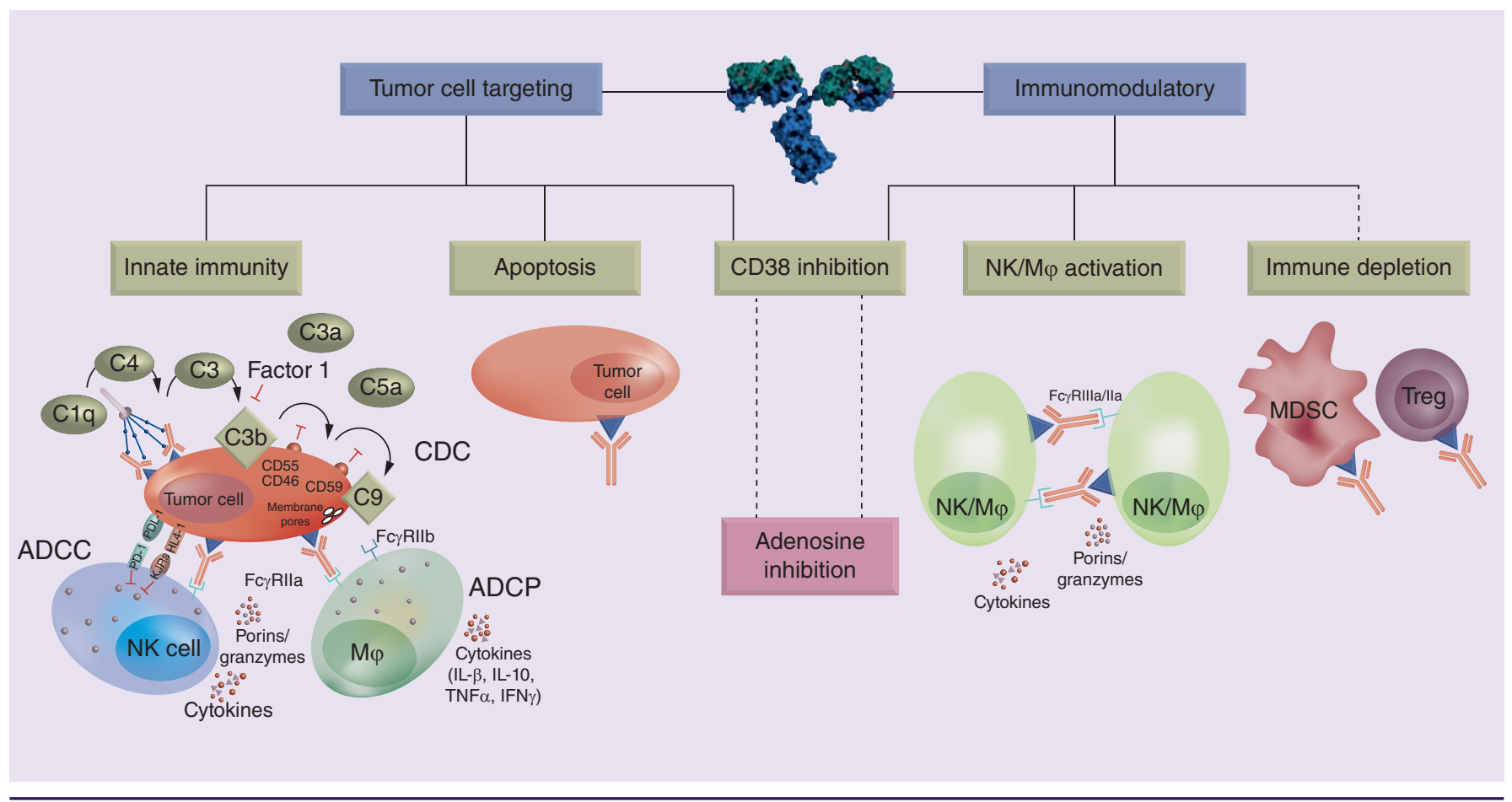

Figure 1. Isatuximab mode of action.

ADCC/CP: Antibody-dependent cellular cytotoxicity/phagocytosis; CDC: Complement-dependent cytotoxicity; M $\varphi$ : Macrophage; MDSC: Myeloid-derived suppressor cell; NK: Natural killer cell; Treg: Regulatory T cell.

The first-in-human, Phase I/II study of ISA as a single agent (NCT01084252) demonstrated clinical activity in a heavily pretreated RRMM population [37,38]. ISA was generally well tolerated in these patients. Infusion-associated reactions (IARs) were the most common adverse event (AE), however the majority of IARs were Grade $1 / 2$ and tended to occur during the first infusion. IARs were clinically manageable, and all recovered without clinical consequences. Premedication to mitigate the incidence and severity of IARs has been used in all ISA studies.

The overall response rate (ORR) at doses $\geq 10 \mathrm{mg} / \mathrm{kg}$ ranged from 20 to $29 \%$ in a heavily pretreated patient population who had received a median of five prior lines of therapy [38]. Median time to first response was 4 weeks and median duration of response was approximately 25 weeks [38].

The single-agent activity of ISA and its favorable safety profile make it a promising candidate for use in combination therapies. The effect of ISA in combination with PIs and IMiDs has been investigated in clinical studies. An ongoing Phase Ib, investigator-sponsored study (NCT02332850) is assessing ISA in combination with carfilzomib in heavily pretreated patients with MM $(n=11)$, and preliminary safety data show an acceptable safety profile. All AEs were grade 1-3 and none resulted in discontinuation of treatment. Grade 1/2 IARs, all of which occurred during the first infusion, were observed in six patients. Overall, six patients achieved a partial response (PR) and two patients achieved a very good partial response (VGPR) [39].

The combination of ISA $10 \mathrm{mg} / \mathrm{kg}$ every 2 weeks with Len/dex (NCT01749969) was also associated with an acceptable safety profile [31]. The most common ISA-related AEs were IARs, which occurred mostly during cycle 1; no IARs were observed after the fourth infusion. Overall, the safety profile of this combination was consistent with the safety profile of single-agent ISA and the combination of Len/dex alone [31,38]. Treatment of this heavily pretreated patient population (median five prior lines) with ISA plus Len/dex resulted in an ORR of 56\%, including 17 (32.7\%) VGPRs and two $(3.8 \%)$ stringent complete responses (sCRs) [31]. The ORRs among patients refractory to Len, or both an IMiD and a PI were $52 \%(\mathrm{n}=42)$ and $45 \%(\mathrm{n}=38)$, respectively. The ORR of the heavily pretreated population ( $\geq 3$ prior lines) was $48 \%(\mathrm{n}=44)$. The pharmacokinetics (PK) of Len was not affected by co-administration with ISA [31]. 
ISA has more recently been assessed in combination with Pom/dex, in a Phase Ib dose-escalation study (NCT02283775). Heavily pretreated patients (median four prior lines) received ISA at doses of 5,10 and $20 \mathrm{mg} / \mathrm{kg}$, in combination with Pom/dex. Overall, 16 patients (62\%) had standard risk and five patients (19\%) had high-risk cytogenetic abnormalities; cytogenetics data were not available for the remaining patients. The median age in this patient population was 65 (42-80) years.

Preliminary data on 26 patients have demonstrated that this combination is clinically active with a manageable safety profile in patients with RRMM [40,41]. The initial infusion rate was $175 \mathrm{mg} / \mathrm{h}$ and the median infusion duration was $3.9 \mathrm{~h}$ for the first infusion and $2.8 \mathrm{~h}$ for subsequent infusions at $10 \mathrm{mg} / \mathrm{kg}$ [40,42]. Overall, IARs occurred in $13(50 \%)$ patients (grade 3 in 1 patient) and were more frequent during the first infusion. The incidence of other AEs was generally consistent with the known safety profile of the individual agents, with a higher but manageable (with dose reduction or delay of Pom and the use of granulocyte colony-stimulating factor) rate of grade 3/4 neutropenia (92\%; grade 4 neutropenic infection in 1 patient). No patient withdrew from the study as a result of neutropenia. Preliminary clinical data showed that overall $65.3 \%$ of 26 evaluable patients achieved at least a PR, including one (3.8\%) sCR, one (3.8\%) complete response (CR), seven (26.9\%) VGPRs and eight (30.8\%) PRs. The clinical benefit rate (at least a minimal response) was $73.0 \%$ [40]. At $10 \mathrm{mg} / \mathrm{kg}$, the ORR was $74.9 \%$ including one (8.3\%) sCR, four (33.3\%) VGPRs and four (33.3\%) PRs. The PK of ISA is unaffected by the co-administration of Pom, such that the dosing of the individual agents does not need to be altered when they are given in combination.

Based on the efficacy and safety data from these studies and PK/pharmacodynamic analyses, the dose of $10 \mathrm{mg} / \mathrm{kg}$ once a week/every 2 weeks was chosen for future combination studies. These encouraging clinical data supported the initiation of a Phase III study of ISA in combination with Pom/dex (ICARIA-MM), the methodology of which is described here.

\section{ICARIA-MM study}

Here we describe the design and rationale for the prospective, multinational, randomized, openlabel, parallel-group, two-arm, Phase III study, ICARIA-MM (NCT02990338), evaluating the efficacy and safety of ISA $10 \mathrm{mg} / \mathrm{kg}$ once a week/every 2 weeks in combination with Pom/dex compared with Pom/dex alone for the treatment of patients with RRMM.

\section{Objectives}

The primary objective of this study is to demonstrate the benefit of ISA in combination with Pom/ dex in the prolongation of progression-free survival (PFS) as compared with Pom/dex alone in patients with RRMM. The key secondary objectives are to evaluate the ORR as per International Myeloma Working Group (IMWG) criteria [43] in each arm, and to compare the overall survival (OS) between the two arms. Other secondary objectives include time to progression, PFS in patients with high-risk cytogenetics (del[17p], $\mathrm{t}[4 ; 14], \mathrm{t}[14 ; 16]$ ), duration of response, safety, ISA PK in combination with Pom, immunogenicity and health-related quality of life (HRQoL).

\section{Key eligibility criteria}

Patients aged $\geq 18$ years should have a documented diagnosis of $M M$ with evidence of measurable disease (serum M-protein $\geq 0.5 \mathrm{~g} / \mathrm{dl}$ and/or urine M-protein $\geq 200 \mathrm{mg} / 24 \mathrm{~h}$ ) and Eastern Cooperative Oncology Group performance status of $\leq 2$. Patients should have received $\geq 2$ prior lines of anti-MM therapy, including $\geq 2$ consecutive cycles of Len and a PI (bortezomib, carfilzomib or ixazomib) given alone or in combination, and have failed treatment on Len and a PI due to progression, refractory disease or intolerance.

Patients should be refractory to the last line of therapy according to IMWG criteria (progression on or within 60 days of the end of the last therapy) [43]. Patients must have adequate liver, renal and BM function, measured as aspartate transaminase and/or alanine transaminase levels $\leq 3 \times$ upper limit of normal, total bilirubin levels $\leq 2 \times$ upper limit of normal, creatinine clearance rate $\geq 30 \mathrm{ml} /$ min, absolute neutrophil count $\geq 1 \times 10^{9} / 1$ and platelet levels of $\geq 75,000$ cells $/ \mu 1$, if $<50 \%$ of BM nucleated cells are plasma cells, and $\geq 30,000$ cells $/ \mu \mathrm{l}$, if $\geq 50 \%$ of BM nucleated cells are plasma cells.

Key exclusion criteria include primary refractory MM (patients who have never achieved at least a minimal response with any treatment during the disease course), free light chain measurable 







\section{CLINICAL TRIAL PROTOCOL Richardson, Attal, Campana et al.}

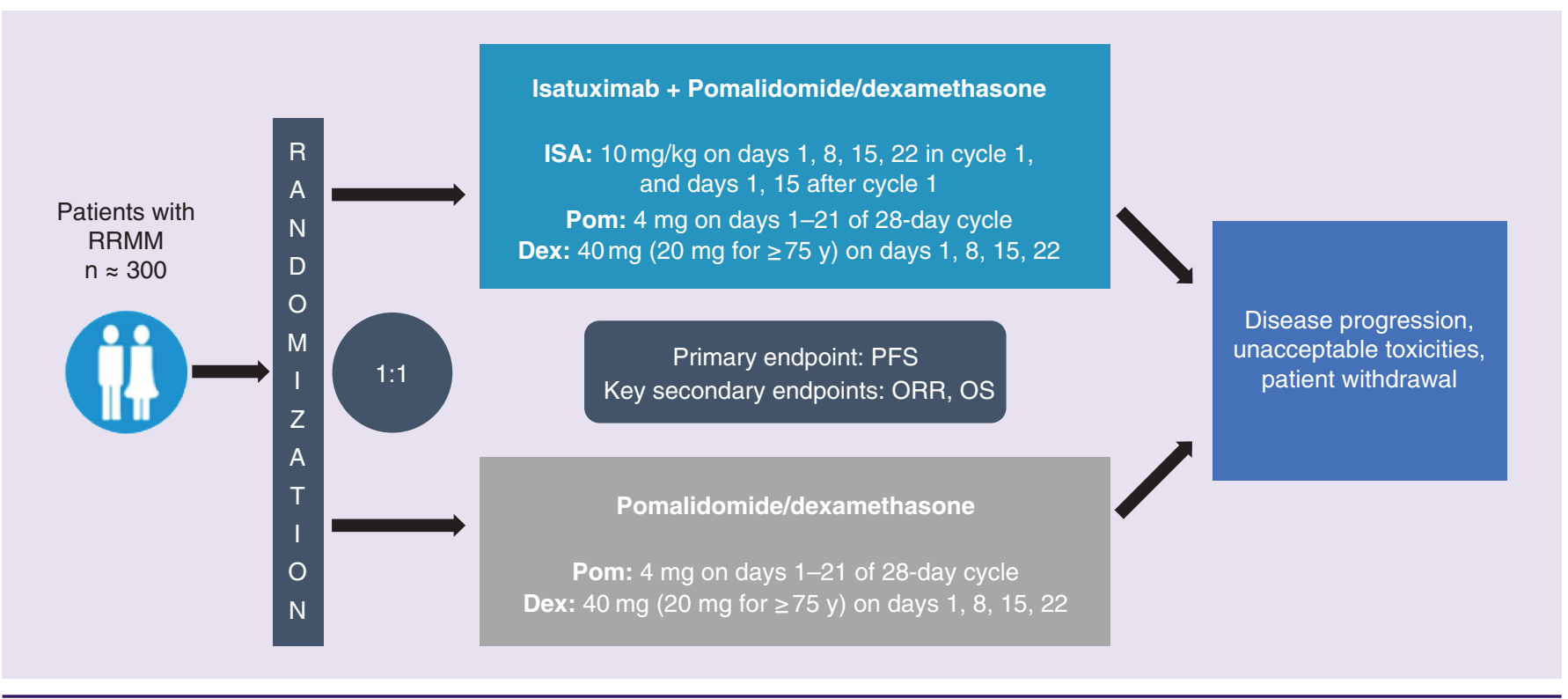

Figure 3. Study design.

Dex: Dexamethasone; ISA: Isatuximab; ORR: Overall response rate; OS; Overall survival; PFS: Progression-free survival;

Pom: Pomalidomide; RRMM: Relapsed and refractory multiple myeloma.

disease only, refractory to prior anti-CD38 mAb treatment, prior Pom therapy and prior allogeneic hematopoietic stem cell transplant with active graft versus host disease (any grade and/or being under immunosuppressive treatment within the last 2 months).

\section{Design}

\section{- Planned study locations}

This Phase III global study is recruiting patients across regions including USA, France, Turkey, Australia, Belgium, Canada, Greece, New Zealand, Norway, Sweden, UK, Denmark, Hungary, Japan, Spain, Taiwan, Czech Republic, Italy, Portugal, Slovakia, Poland, Germany, Korea and Russia (Figure 2).

\section{- Study design \& methodology}

Eligible patients will be randomly assigned in a 1:1 ratio to receive either ISA in combination with Pom/dex or Pom/dex (Figure 3). Treatment is to be given in 28-day cycles, with administration as follows: ISA $10 \mathrm{mg} / \mathrm{kg}$ on days $1,8,15$ and 22 at cycle 1 and days 1 and 15 for subsequent cycles; Pom $4 \mathrm{mg}$ will be administered on days 1-21 of each cycle; dex $40 \mathrm{mg}$ (or $20 \mathrm{mg}$ if the patient is $\geq 75$ years old) will be administered on days $1,8,15$ and 22 of each cycle.

Patients allocated to receive ISA will receive routine premedications prior to ISA infusion to reduce the risk and severity of IARs, which are commonly observed with mAbs. Premedication consists of diphenhydramine at 25-50 mg iv, ranitidine at $50 \mathrm{mg}$ iv and acetaminophen at 650-1000 $\mathrm{mg}$ or equivalents of each. Dex will be given prior to ISA infusion as part of premedication and part of study treatment. Patients will continue treatment until disease progression, unacceptable AEs or patient preference.

Randomization will be stratified by age ( $<75$ years vs $\geq 75$ years) and number of previous lines of therapy ( 2 or 3 vs $>3)$.

\section{- Outcome measures/endpoints}

Demographic characteristics and medical history, prior MM history (time from diagnosis, stage at diagnosis/study entry, prior anti-MM therapies) and BM samples were collected at baseline.

Evaluation of response and disease progression will be determined according to IMWG criteria [43] based on an Independent Response Review Committee, on day 1 of each cycle and at the end of treatment. The primary efficacy endpoint will be PFS (the time from the date of randomization to 
Table 1. Outcome assessments and schedule.

\begin{tabular}{|c|c|c|}
\hline \multirow{2}{*}{$\begin{array}{l}\text { Patient characteristics } \\
\text { and treatment history }\end{array}$} & Timing & Assessment \\
\hline & Baseline & $\begin{array}{l}\text { Patient demographics } \\
\text { Prior MM history }\end{array}$ \\
\hline Disease response $^{\dagger}$ & D1 of all cycles, EOT & $\begin{array}{l}\text { Serum M-protein (SPEP, IFX) } \\
\text { Urine M-protein (UPEP, IFX) } \\
\text { Serum-free light chains (Freelite) } \\
\text { Quantitative immunoglobulins } \\
\text { Bone marrow aspirate }{ }^{\S} \\
\text { Bone disease assessment (skeletal survey or low-dose whole-body CT scan) } \\
\text { Extramedullary disease assessment } \\
\text { (CT scan or MRI)ף }\end{array}$ \\
\hline Safety & $\begin{array}{l}\text { Continuously throughout } \\
\text { study period }\end{array}$ & $\begin{array}{l}\text { Adverse events } \\
\text { Vital signs } \\
\text { Physical examinations } \\
\text { Laboratory abnormalities } \\
\text { Hematology } \\
\text { Blood chemistry } \\
\text { Antidrug antibodies } \\
\text { Any other relevant examinations if clinically indicated }\end{array}$ \\
\hline $\begin{array}{l}\text { Isatuximab } \\
\text { pharmacokinetics }\end{array}$ & $\begin{array}{l}\text { Cycle 1: D1, D8, D15, D22 } \\
\text { Subsequent: D1, D15, EOT }\end{array}$ & $\begin{array}{l}\text { Isatuximab PK parameters by ELISA } \\
\text { Population PK modeling }\end{array}$ \\
\hline $\begin{array}{l}\text { Patient-reported } \\
\text { outcome }\end{array}$ & $\begin{array}{l}\text { D1 of all cycles } 30 \text { and } 60 \\
\text { days post treatment }\end{array}$ & $\begin{array}{l}\text { Electronic questionnaire: } \\
\text { QLQ-C30, MY20, EQ-5D-5L }\end{array}$ \\
\hline $\begin{array}{l}\text { 'Responses will be assessed ac } \\
\text { "For patients who discontinue } \\
\text { 'Bone marrow assessment at b } \\
\text { 'In cases of known/documente } \\
\text { D: Day; EOT: End of treatment; } \\
\text { PK: Pharmacokinetic; QLQ-C30 }\end{array}$ & $\begin{array}{l}\text { Cording to the International Myelo } \\
\text { reatment without disease progre } \\
\text { aseline and any time during the st } \\
\text { d extramedullary disease at basel } \\
\text { QQ-5D-5L: EuroQoL 5-Dimensions } \\
\text { Quality of life questionnaire-C30; }\end{array}$ & $\begin{array}{l}\text { Working Group criteria based on an independent response review committee. } \\
\text { n, disease response will be assessed every month during follow-up until disease progression. } \\
\text { as clinically indicated. } \\
\text { assessment will be performed at baseline and every } 12 \text { weeks, and if clinically indicated. } \\
\text { evels questionnaire; IFX: Immunofixation; MM: Multiple myeloma; MY20: Quality of life questionnaire MY20; } \\
\text { P: Serum protein electrophoresis; UPEP: Urine protein electrophoresis. }\end{array}$ \\
\hline
\end{tabular}

the date of first documentation of progressive disease or the date of death from any cause, whichever comes first). For patients who discontinue treatment without disease progression, disease response will be assessed every month during follow-up until disease progression. Minimal residual disease will be assessed by next-generation sequencing using BM aspirates collected at baseline and when a CR is confirmed. If patients achieve a CR but are minimal residual disease positive, subsequent tests will be performed 3 and 6 months later to identify a late response.

Safety evaluations including AEs, laboratory data and vital signs will be collected from receipt of informed consent to at least 30 days after the last dose. Any ongoing serious AEs or treatmentrelated AEs at that time will be followed until recovery or stabilization. AEs will be graded using National Cancer Institute-Common Terminology Criteria for Adverse Events (NCI-CTCAE) v4.03. An indirect Coombs' test will be performed at baseline and during treatment. Immunogenicity will be assessed during study treatment.

PK evaluations will include an estimation of interpatient PK variability, population PK parameter estimates and the assessment of pathophysiologic covariate effects on volume.

Patient-reported outcome (PRO) assessments will be measured on day 1 of all cycles and at the end of treatment using the PRO/HRQoL and health utility instruments (European Organization for Research and Treatment of Cancer-quality of life questionnaires C-30 and MY20, and the EuroQoL questionnaire EQ-5D-5L).

The assessment schedule for all measures is described in Table 1.

\section{Statistical considerations}

\section{- Analysis set}

The intent-to-treat (ITT) population will include all patients who have given informed consent and 
for whom there is confirmation of successful allocation of a randomization number by the interactive response technology. Analyses using this population will be based on the treatment arm as randomized, regardless of whether patients receive any study drug or receive a different study drug from which they were originally randomized. This population will be the primary population for all efficacy parameters.

The safety population will include ITT patients who receive at least one dose or part of a dose of the study treatments. This population is the primary population for all safety analysis parameters. All analyses using this population will be based on the treatment actually received.

The PK population will include patients from the safety population who receive at least one dose of ISA, even if incomplete, with data for at least one PK parameter available. PROs will be assessed in patients from the safety population who have completed the baseline and at least one postbaseline assessment for at least one of the three selected PRO/HRQoL and health utility instruments.

\section{- Statistical analysis}

Analysis for the primary endpoint, PFS, will be performed on the ITT population, with comparison using a log-rank test procedure at the one-sided level of 0.025 stratified by stratification factors as entered in the interactive response technology (i.e., age and number of previous lines of therapy).

Safety analysis will be descriptive. PK data available from Phase I, II and III studies will be used for the analysis. The population estimates from this analysis will provide a prior distribution from which individual Bayesian estimates of the PK parameters for each patient in this study will be derived. PK parameters will be summarized by descriptive statistics, such as mean, geometric mean, median, standard deviation, standard error of the mean, minimum and maximum.

\section{- Sample size}

A maximum of 300 patients (150 patients in each arm, randomized 1:1) will be enrolled in the study. Assuming proportional hazards, a total of 162 PFS events will be needed to detect a hazard ratio of 0.6 using a log-rank test at the one-sided 0.025 level with a $90 \%$ power.

\section{Discussion}

Significant improvements have been made in the treatment of MM, yet there remains an unmet medical need for patients with RRMM. Although the development of novel agents has improved outcomes, the majority of patients with MM will eventually relapse, and patients who have failed the current standard of care treatments have a poor prognosis [19]. Outcomes are generally worse for patients who are refractory to a number of agents, with a lower OS in patients who have become refractory to both PIs and IMiDs $[19,20,44]$.

This manuscript describes the study design of the pivotal ISA ICARIA-MM Phase III study, which will investigate ISA in combination with Pom/dex, and the effect on PFS in patients with RRMM. The effect of Pom on the immune system coupled with the mechanism of action of ISA and preclinical data demonstrating significantly enhanced direct and indirect anti-MM cytotoxicity when ISA is combined with Pom [36] suggest that the combination of both drugs may improve the benefit seen with Pom/dex alone in clinical studies of patients with RRMM (median PFS: 4.0 months; median OS: 13.1 months) [45]. ISA has been investigated at three dose levels (5, 10 and $20 \mathrm{mg} / \mathrm{kg})$ in combination with Pom/dex in a Phase Ib study of patients with RRMM $(\mathrm{n}=26)$ treated with $\geq 2$ previous lines of therapy and revealed promising results in terms of ORR and the safety profile [46]. The ICARIA-MM Phase III study will evaluate the clinical benefit of ISA $10 \mathrm{mg} /$ $\mathrm{kg}$ in combination with Pom/dex compared with Pom/dex alone, in a larger patient population than the Phase Ib study (approximately 300 patients) using a randomized, controlled study design.

Eligible patients will have failed at least two prior therapies including Len and a PI. This patient population is particularly difficult to treat. Examination of 286 patients refractory to bortezomib and relapsed, refractory or intolerant to an IMiD (Len or thalidomide) demonstrated a median event-free survival of 5 months and a median OS of 9 months [19]. The presence of at least one highrisk cytogenetic abnormality was also associated with low OS compared with patients with standard risk cytogenetics (7 months vs 17 months) [19]. Therapy options in these patients are limited, and therefore new treatments which improve outcomes and survival are required. ISA has previously 
Table 2. Completed, ongoing and future studies of isatuximab for the treatment of multiple myeloma.

\begin{tabular}{|c|c|c|c|}
\hline NCT number & Combination & Status & Patient population \\
\hline \multicolumn{4}{|c|}{ Phase I or Phase I/II } \\
\hline $\begin{array}{l}\text { NCT01749969 } \\
\text { Phase I/II }\end{array}$ & ISA, Len, dex & Completed & $\mathrm{RRMM}, \geq 2$ prior lines of therapy including IMiD \\
\hline $\begin{array}{l}\text { NCT01084252 } \\
\text { Phase I/II }\end{array}$ & ISA, dex & $\begin{array}{l}\text { Phase I and Phase II stage } 1 \\
\text { completed, } \\
\text { Phase II stage } 2 \text { recruiting }\end{array}$ & RRMM, progression after standard therapy including IMiD or PI \\
\hline $\begin{array}{l}\text { NCT02514668 } \\
\text { Phase I }\end{array}$ & ISA & Recruiting & $\begin{array}{l}\text { RRMM, } \geq 3 \text { prior lines of therapy including IMiD (for } \geq 2 \text { cycles or } \\
\geq 2 \text { months) and } \mathrm{PI} \text { (for } \geq 2 \text { cycles or } \geq 2 \text { months) }\end{array}$ \\
\hline $\begin{array}{l}\text { NCT02513186 } \\
\text { Phase I }\end{array}$ & $\begin{array}{l}\text { ISA + VCD } \\
\text { or } \\
\text { ISA + VRD }\end{array}$ & $\begin{array}{l}\text { ISA + VCD: } \\
\text { active not recruiting } \\
\text { ISA + VRD: } \\
\text { recruiting }\end{array}$ & Newly diagnosed MM \\
\hline \multicolumn{4}{|l|}{ Phase II } \\
\hline $\begin{array}{l}\text { NCT02960555 } \\
\text { Phase II }\end{array}$ & ISA & Recruiting & Smoldering MM \\
\hline $\begin{array}{l}\text { NCT03104842 } \\
\text { Phase II }\end{array}$ & $\begin{array}{l}\text { ISA, Car, Len, } \\
\text { dex }\end{array}$ & Recruiting & Newly diagnosed MM \\
\hline \multicolumn{4}{|l|}{ Phase III } \\
\hline $\begin{array}{l}\text { NCT03275285 } \\
\text { Phase III }\end{array}$ & $\begin{array}{l}\text { ISA, Car, dex } \\
\text { or } \\
\text { Car, dex }\end{array}$ & Recruiting & RRMM, 1-3 prior lines of therapy \\
\hline
\end{tabular}

demonstrated anti-MM activity in patients who are refractory to currently available IMiDs and/ or PIs, and those with high-risk cytogenetic abnormalities [31,39,46]. At the dose selected for the ICARIA-MM study (10 mg/kg once a week/every 2 weeks), ISA in combination with Pom/dex showed clinical efficacy in a heavily pretreated population [44]. The promising response rates also observed among patients refractory to Len, PIs, or IMiDs and PIs suggest that the combination of ISA and Pom/dex may be a suitable therapy for these patient populations [46]. Pom has previously shown efficacy in patients who were refractory to Len and also patients who were refractory to both Len and bortezomib $[8,47]$. Pom/dex has also shown encouraging data in patients with high-risk cytogenetic abnormalities who have a poor prognosis [48].

The key endpoints of the ICARIA-MM study are PFS (primary endpoint), ORR and OS (secondary endpoints). PFS is commonly used as a primary endpoint in Phase III studies in RRMM, and improvements in PFS have previously been demonstrated in clinical studies of patients with RRMM treated with $\mathrm{mAbs}$ in combination with IMiDs [25,49]. ELO in combination with Len/dex demonstrated an improvement in PFS, with a difference in median PFS of 4.5 months, compared with Len/dex alone (19.4 vs 14.9 months) [25]. Similarly, median PFS was longer in patients receiving DARA in combination with Len/dex compared with Len/dex alone (not reached vs 18.4 months) [49].

ISA and DARA are both anti-CD38 therapies, however their modes of action differ, which may translate into differences in clinical activity. These agents bind to different unique epitopes on CD38, and ISA is the only anti-CD38 mAb with demonstrated direct apoptotic activity [22]. The 
shorter infusion time of ISA may also represent an advantage for patient convenience compared with DARA. In addition, DARA has recently been approved by the US FDA as a second-line treatment for RRMM, in combination with Len/dex or bortezomib/dex [50].

The ICARIA-MM study is the first study to investigate the efficacy of ISA in RRMM in a randomized Phase III trial. In addition to this current study, ongoing studies are investigating ISA as an add-on therapy to current standard-of-care regimens in RRMM, newly diagnosed MM and smoldering MM (Table 2).

\author{
Acknowledgements \\ The authors would like to acknowledge J San Miguel and V Rajkumar for their input into the design of the study as \\ members of the Phase III Steering Committee and S Micallef for valuable statistical support during protocol \\ development.
Financial \& competing interests disclosure
This study is sponsored by Sanofi. PG Richardson was a part of consulting activity for Genmab, research funding from Celgene. F Campana, M-L Risse, S Le-Guennec and K Corzo are employees of Sanofi. AM Hui was former employee of Sanofi. MAttal and KC Anderson have no disclosures to report. The authors have no other relevant affiliations or financial involvement with any organization or entity with a financial interest in or financial conflict with the subject matter or materials discussed in the manuscript apart from those disclosed.
Editorial support was provided by L Wright, PhD, of Adelphi Communications Ltd, funded by Sanofi.

\title{
Executive summary
}

\section{Multiple myeloma}

- Multiple myeloma ( $\mathrm{MM})$ is a malignant plasma cell disease, characterized by proliferation of plasma cells in the bone marrow and the production of excessive amounts of a monoclonal immunoglobulin. MM is considered largely incurable, although survival rates have improved over recent years, and most patients will ultimately relapse following therapy. Therefore, treatment for relapsed/refractory multiple myeloma (RRMM) remains an unmet need.

\section{Isatuximab}

- Isatuximab is a monoclonal antibody that binds selectively to a unique epitope on CD38, and targets myeloma cells through a combination of mechanisms. Isatuximab has shown efficacy and tolerability as a monotherapy and combination therapy, with immunomodulatory drugs such as lenalidomide and pomalidomide (Pom) in Phase I/II studies in heavily pretreated patients with RRMM.

\section{ICARIA-MM}

- Here, we describe the design of the Phase III ICARIA-MM study (NCT02990338) which will evaluate isatuximab in combination with Pom and low-dose dexamethasone (dex) (Pom/dex) versus Pom/dex alone in RRMM.

\section{Objectives}

- The primary objective of the ICARIA-MM study is to demonstrate the benefit of isatuximab in combination with Pom/dex in the prolongation of progression-free survival (PFS) compared with Pom/dex alone in patients with RRMM. The secondary objectives include evaluating overall response rate as per International Myeloma Working Group criteria and to compare overall survival between dosing groups.

\section{Key eligibility}

- Eligible patients are adults with RRMM and confirmed disease progression within 60 days of the last therapy, and who have received at least two prior lines of therapy, including lenalidomide and a proteasome inhibitor (bortezomib, carfilzomib or ixazomib) alone or in combination.

\section{Study design}

- Patients will be randomized in a 1:1 ratio to receive isatuximab plus Pom/dex or Pom/dex alone in 28-day cycles. Patients will continue therapy until progression, unacceptable toxicity or patient/investigator decision to discontinue the study.

\section{Statistical considerations}

- The primary endpoint will be PFS. The intent-to-treat (ITT) population will be the primary population for all efficacy parameters. Analysis for PFS will be performed on the ITT population, with comparison using a log-rank test procedure, at the one-sided level of 0.025 . The safety population will include ITT patients who receive at least part of a dose of the study treatments. Safety evaluations will include adverse events/serious adverse events, laboratory parameters, vital signs and physical examination.

- Approximately 300 patients (150 in each arm) are expected to enroll. The first patient was recruited in January 2017 and accrual is ongoing. 


\section{Open access}

This work is licensed under the Attribution-NonCommercial-NoDerivatives 4.0 Unported License. To view a copy of this license, visit http://creativecommons. org/licenses/by-nc-nd/4.0/

\section{References}

Papers of special note have been highlighted as: $\bullet$ of interest; $\bullet \bullet$ of considerable interest

1 Rollig C, Knop S, Bornhauser M. Multiple myeloma. Lancet 385(9983), 2197-2208 (2015).

2 Binder M, Rajkumar SV, Ketterling RP et al. Occurrence and prognostic significance of cytogenetic evolution in patients with multiple myeloma. Blood Cancer J. 6, e401 (2016).

3 Sonneveld P, Avet-Loiseau H, Lonial S et al. Treatment of multiple myeloma with high-risk cytogenetics: a consensus of the International Myeloma Working Group. Blood 127(24), 2955-2962 (2016).

4 Hari P. Recent advances in understanding multiple myeloma. Hematol. Oncol. Stem Cell Ther. doi:10.1016/j.hemonc.2017.05.005 (2017) (Epub ahead of print).

5 Eshaghian S, Berenson JR. Multiple myeloma: improved outcomes with new therapeutic approaches. Curr. Opin. Support. Palliat. Care 6(3), 330-336 (2012).

6 Stewart AK, Rajkumar SV, Dimopoulos MA et al. Carfilzomib, lenalidomide, and dexamethasone for relapsed multiple myeloma. N. Engl. J. Med. 372(2), $142-152$ (2015).

7 Moreau P, Masszi T, Grzasko N et al. Oral ixazomib, lenalidomide, and dexamethasone for multiple myeloma. N. Engl. J. Med. 374(17), 1621-1634 (2016).

8 San Miguel J, Weisel K, Moreau P et al. Pomalidomide plus low-dose dexamethasone versus high-dose dexamethasone alone for patients with relapsed and refractory multiple myeloma (MM-003): a randomised, open-label, Phase III trial. Lancet Oncol. 14(11), 1055-1066 (2013).

9 Richardson PG, Siegel DS, Vij R et al. Pomalidomide alone or in combination with low-dose dexamethasone in relapsed and refractory multiple myeloma: a randomized Phase II study. Blood 123(12), 1826-1832 (2014).

10 Morgan GJ, Davies FE, Gregory WM et al. Effects of induction and maintenance plus long-term bisphosphonates on bone disease in patients with multiple myeloma: the Medical Research Council Myeloma IX Trial. Blood 119(23), 5374-5383 (2012).

11 Niesvizky R, Martin TG 3rd, Bensinger WI et al. Phase Ib dose-escalation study (PX-171-006) of carfilzomib, lenalidomide, and low-dose dexamethasone in relapsed or progressive multiple myeloma. Clin. Cancer Res. 19(8), 2248-2256 (2013).

12 Jakubowiak AJ, Dytfeld D, Griffith KA et al. A Phase 1/2 study of carfilzomib in combination with lenalidomide and low-dose dexamethasone as a frontline treatment for multiple myeloma. Blood 120 (9), 1801-1809 (2012).

13 Richardson PG, Weller E, Lonial S et al. Lenalidomide, bortezomib, and dexamethasone combination therapy in patients with newly diagnosed multiple myeloma. Blood 116(5), 679-686 (2010).

14 Quach H, Ritchie D, Stewart AK et al. Mechanism of action of immunomodulatory drugs (IMiDS) in multiple myeloma. Leukemia 24(1), 22-32 (2010).

15 Holstein SA, Mccarthy PL. Immunomodulatory drugs in multiple myeloma: mechanisms of action and clinical experience. Drugs 77(5), 505-520 (2017)

16 Dimopoulos MA, Swern AS, Li JS et al. Efficacy and safety of long-term treatment with lenalidomide and dexamethasone in patients with relapsed/ refractory multiple myeloma. Blood Cancer J. 4, e257 (2014).

17 Palumbo A, Rajkumar SV, San Miguel JF et al. International Myeloma Working Group consensus statement for the management, treatment, and supportive care of patients with myeloma not eligible for standard autologous stem-cell transplantation. J. Clin. Oncol. 32(6), 587-600 (2014).

18 Richardson PG, Weller E, Jagannath S et al. Multicenter, Phase I, dose-escalation trial of lenalidomide plus bortezomib for relapsed and relapsed/refractory multiple myeloma. J. Clin. Oncol. 27(34), 5713-5719 (2009).

19 Kumar SK, Lee JH, Lahuerta JJ et al. Risk of progression and survival in multiple myeloma relapsing after therapy with IMiDs and bortezomib: a multicenter international myeloma working group study. Leukemia 26(1), 149-157 (2012).

20 Laubach J, Garderet L, Mahindra A et al. Management of relapsed multiple myeloma: recommendations of the International Myeloma Working Group. Leukemia 30(5), 1005-1017 (2016).

21 Lonial S, Dimopoulos M, Palumbo A et al. Elotuzumab therapy for relapsed or refractory multiple myeloma. N. Engl. J. Med. 373(7), 621-631 (2015).

22 Deckert J, Wetzel MC, Bartle LM et al. SAR650984, a novel humanized CD38-targeting antibody, demonstrates potent antitumor activity in models of multiple myeloma and other CD38+ hematologic malignancies. Clin. Cancer Res. 20(17), 4574-4583 (2014).

- A preclinical study reporting the mechanisms of action of isatuximab (ISA), highlighting the potent antitumor activity of this agent in cell lines and mouse models of multiple myeloma and other $\mathrm{CD} 8^{+}$hematologic malignancies.

23 Lonial S, Weiss BM, Usmani SZ et al. Daratumumab monotherapy in patients with treatment-refractory multiple myeloma (SIRIUS): an open-label, randomised, Phase II trial. Lancet 387(10027), 1551-1560 (2016).

24 Lokhorst HM, Plesner T, Laubach JP et al. Targeting CD38 with daratumumab monotherapy in multiple myeloma. N. Engl. J. Med. 373(13), 1207-1219 (2015). 


\section{CLINICAL TRIAL PROTOCOL Richardson, Attal, Campana et al.}

25 Richardson PG, Jagannath S, Moreau P et al. Elotuzumab in combination with lenalidomide and dexamethasone in patients with relapsed multiple myeloma: final Phase II results from the randomised, open-label, Phase Ib-II dose-escalation study. Lancet Haematol. 2(12), e516-e527 (2015).

26 Malavasi F, Deaglio S, Funaro A et al. Evolution and function of the ADP ribosyl cyclase/CD38 gene family in physiology and pathology. Physiol. Rev. 88(3), 841-886 (2008).

27 Chari A, Suvannasankha A, Fay JW et al. Daratumumab plus pomalidomide and dexamethasone in relapsed and/or refractory multiple myeloma. Blood 130(8), 974-981 (2017).

28 Jelinek T, Hajek R. Monoclonal antibodies - a new era in the treatment of multiple myeloma. Blood Rev. 30(2), 101-110 (2016).

29 Feng X, Zhang L, Acharya C et al. Targeting CD38 suppresses induction and function of T regulatory cells to mitigate immunosuppression in multiple myeloma. Clin. Cancer Res. 23(15), 4290-4300 (2017).

- A preclinical study reporting the effect of ISA on immunosuppressive regulatory T cells. ISA treatment may restore the immune function against multiple myeloma cells.

30 Moreno L, Zabaleta A, Alignani D et al. Critical analysis on the mechanism of action (MoA) of the anti-CD38 monoclonal antibody isatuximab in multiple myeloma (MM). Presented at: 58th ASH Annual Meeting \& Exposition. CA, USA, 3-6 December 2016.

31 Martin T, Baz R, Benson DM et al. A Phase Ib study of isatuximab plus lenalidomide and dexamethasone for relapsed/refractory multiple myeloma. Blood 129(25), 3294-3303 (2017).

- A Phase Ib study reporting the effect of ISA in combination with lenalidomide (Len)/dexamethasone (dex). ISA in combination with Len/dex was tolerated and was active in heavily pretreated patients with relapsed/refractory multiple myeloma (RRMM).

32 Zhang B, Yang G, Dai S et al. SAR650984, a humanized anti-CD38 antibody potently modulates intracellular and extracellular nucleotide levels of cancer cells. Presented at: 105th Annual Meeting of the American Association for Cancer Research. CA, USA, 5-9 April 2014.

33 Wetzel M-C, Nicolazzi C, Vallée F et al. SAR650984: characterization of a potent Phase I humanized anti-CD38 antibody for the treatment of multiple myeloma and other hematologic malignancies. Presented at: American Association for Cancer Research 104th Annual Meeting. DC, USA, 6-10 April 2013.

34 Shen LS, Wang J, Shen DF et al. CD4(+)CD25(+)CD127(low/-) regulatory T cells express Foxp3 and suppress effector T cell proliferation and contribute to gastric cancers progression. Clin. Immunol. 131(1), 109-118 (2009).

35 Wolf AM, Wolf D, Steurer M, Gastl G, Gunsilius E, Grubeck-Loebenstein B. Increase of regulatory T cells in the peripheral blood of cancer patients. Clin. Cancer Res. 9(2), 606-612 (2003).

36 Jiang H, Acharya C, An G et al. SAR650984 directly induces multiple myeloma cell death via lysosomal-associated and apoptotic pathways, which is further enhanced by pomalidomide. Leukemia 30 (2), 399-408 (2016).

-• A preclinical study reporting the enhanced effect of pomalidomide (Pom) on the activity of ISA, in Pom- and Len-resistant cells. This study highlights the preclinical evidence supporting therapies combining ISA with immunomodulatory drugs.

37 Martin TG, Hsu K, Strickland SA, Glenn MJ, Mikhael J, Charpentier E. A Phase I trial of SAR650984, a CD38 monoclonal antibody, in relapsed or refractory multiple myeloma. Presented at: American Society of Clinical Oncology 50th Annual Meeting. IL, USA, 30 May-3 June 2014.

38 Richter JR, Martin TG, Vij R et al. Updated data from a Phase II dose finding trial of single agent isatuximab (SAR650984, anti-CD38 mAb) in relapsed/ refractory multiple myeloma (RRMM). Presented at: The American Society of Clinical Oncology 52nd Annual Meeting. IL, USA, 3-7 June 2016.

-• A Phase II study reporting the effect of ISA monotherapy. ISA monotherapy was active and tolerated in heavily pretreated patients with RRMM.

39 Martin TG, Mannis GN, Chari A et al. Phase Ib study of isatuximab and carfilzomib in relapse and refractory multiple myeloma. Presented at: American Society of Hematology 58th Annual Meeting \& Exposition. CA, USA, 3-6 December 2016.

40 Mikhael J, Richardson PG, Usmani SZ et al. A Phase Ib study of isatuximab in combination with pomalidomide (Pom) and dexamethasone (Dex) in relapsed/refractory multiple myeloma (RRMM). Presented at: American Society of Clinical Oncology 2017 Annual Meeting. IL, USA, 2-6 June 2017.

41 Richardson P, Mikhael J, Usmani S et al. Preliminary results from a Phase $1 \mathrm{~b}$ study of isatuximab in combination with pomalidomide (Pom) and dexamethasone (dex) in relapsed/refractory multiple myeloma. Presented at: 16th International Myeloma Workshop 2017. New Delhi, India, 1-4 March 2017.

42 Richardson PG, Mikhael J, Usmani SZ et al. Preliminary results from a Phase Ib study of isatuximab in combination with pomalidomide and dexamethasone in relapsed and refractory multiple myeloma. Presented at: American Society of Hematology 58th Annual Meeting \& Exposition. CA, USA, 3-6 December 2016.

43 Kumar S, Paiva B, Anderson KC et al. International Myeloma Working Group consensus criteria for response and minimal residual disease assessment in multiple myeloma. Lancet Oncol. 17(8), e328-e346 (2016).

44 Usmani S, Ahmadi T, Ng Y et al. Analysis of real-world data on overall survival in multiple myeloma patients with $\geq 3$ prior lines of therapy including a proteasome inhibitor (PI) and an immunomodulatory drug (IMiD), or double refractory to a PI and an IMiD. Oncologist 21(ii), 1355-1361 (2016).

45 San Miguel JF, Weisel KC, Song KW et al. Impact of prior treatment and depth of response on survival in MM-003, a randomized Phase 3 study comparing pomalidomide plus low-dose dexamethasone versus high-dose dexamethasone in relapsed/refractory multiple myeloma. Haematologica 100(10), 1334-1339 (2015).

46 Mikhael J, Richardson P, Usmani S et al. A Phase Ib study of isatuximab plus pomalidomide (Pom) and dexamethasone (dex) in relapsed/refractory multiple myeloma (RRMM). Presented at: 22nd Congress of the European Hematology Association Madrid, Spain, 22-25 June 2017.

- A Phase Ib study reporting the effect of ISA in combination with Pom/dex. ISA combined with Pom/dex was manageable and clinically active in 
heavily pretreated patients with RRMM.

47 Vij R, Richardson PGG, Jagannath S et al. Pomalidomide (POM) with or without low-dose dexamethasone (LoDEX) in patients (pts) with relapsed/ refractory multiple myeloma (RRMM): outcomes in pts refractory to lenalidomide (LEN) and/or bortezomib (BORT). J. Clin. Oncol. 30 (15 Suppl.), 8016 (2012).

48 Leleu X, Karlin L, Macro M et al. Pomalidomide plus low-dose dexamethasone in multiple myeloma with deletion 17p and/or translocation (4;14): IFM 2010-02 trial results. Blood 125(9), 1411-1417 (2015).

49 Dimopoulos MA, Oriol A, Nahi H et al. Daratumumab, lenalidomide, and dexamethasone for multiple myeloma. N. Engl. J. Med. 375(14), 1319-1331 (2016).

50 Bhatnagar V, Gormley NJ, Luo L et al. FDA approval summary: daratumumab for treatment of multiple myeloma after one prior therapy. Oncologist 22(11), 1347-1353 (2017). 